\title{
New mechanism of cluster-field evaporation in rf breakdown
}

\author{
Z. Insepov, J. H. Norem, and A. Hassanein \\ Argonne National Laboratory, 9700 South Cass Avenue, Argonne, Illinois 60439, USA
}

(Received 26 April 2004; published 22 December 2004)

\begin{abstract}
Using a simple field evaporation model and molecular dynamics simulations of nanoscale copper tip evolution in a high electric field gradient typical for linacs, we have studied a new mechanism for rf-field evaporation. The mechanism consists of simultaneous (collective) field evaporation of a large group of tip atoms in high-gradient fields. Thus, evaporation of large clusters is energetically more favorable when compared with the conventional, "one-by-one" mechanism. The studied mechanism could also be considered a new mechanism for the triggering of rf-vacuum breakdown. This paper discusses the mechanism and the experimental data available for electric field evaporation of field-emission microscopy tips.
\end{abstract}

DOI: 10.1103/PhysRevSTAB.7.122001

PACS numbers: 29.17.+w, 52.80.Vp, 68.37.Vj

\section{INTRODUCTION}

Higher voltage gradients are required for development of future $\mathrm{TeV}$ linear accelerators and muon-muon colliders. However, the development of these accelerators and colliders is seriously hampered by the breakdown of electric vacuum between the electrodes in the accelerator structures, power sources, and waveguides.

Electrical breakdown in vacuum, for both $\mathrm{rf}$ and $\mathrm{dc}$ systems, has been studied for many years, and many mechanisms have been proposed to explain this phenomenon [1-4]. Breakdown seems to have a sharp threshold in electric field, but somewhat weak dependence on frequency, temperature of structure, or vacuum conditions $[5,6]$.

Recent experiments have shown that breakdown occurs when the tensile stress exerted by local electric fields becomes comparable to the tensile strength of the metal [6]. While single atoms may not interact strongly with the electric fields, the emission of clusters and fragments, in the presence of field emitted electron beams, may produce very high local power densities $\left(10^{13}-10^{14} \mathrm{~W} / \mathrm{cm}^{3}\right)$, which could rapidly ionize and damage the structure [7].

This paper describes the emission of clusters under conditions existing in cavities and shows how they can be emitted and accelerated by the electric fields. This new mechanism was studied using a molecular dynamics (MD) simulation of a nanoscale copper tip on an interior surface of an rf cavity [8]. The simulation describes the surface conditions and estimates the temperature and electric field dependence of this mechanism.

\section{DIRECT CURRENT FIELD EVAPORATION}

Direct current electric field evaporation (DCFE) of single ions from a metal tip is a well-known phenomenon and is a key element for imaging by field ion microscopy (FIM) techniques [9-13] and by scanning tunneling microscopy tips [14-18].
Experimental observation of field evaporation takes place in dc electric fields $10 \mathrm{GV} / \mathrm{m}$. The temperature of the FIM tip is set to be sufficiently low to eliminate ordinary thermal evaporation, which takes place at higher temperatures close to the melting point of the tip material. A simplified model of field evaporation, applicable to $0 \mathrm{~K}$, was developed by Müller; it gives the following formula for the threshold electric field above which field evaporation becomes visible [9-12]:

$$
F_{\mathrm{ev}}=\frac{1}{Z r_{0}}\left(\Lambda+\sum_{i}^{n} I_{i}-Z \phi-\frac{3.6 Z^{2}}{r_{0}}\right) \mathrm{V} / \AA .
$$

Here $Z$ is the charge state of an evaporating atom $(Z=1$ for a single charge), $r_{0}$ is the distance of the atom from an ideal flat surface, $\Lambda$ is the binding energy of a neutral atom, $I_{i}$ represents the ionization energies for different charge states of an atom, and $\phi$ is the work function of the material. All energy variables that enter Eq. (1) are in $\mathrm{eV}$ and the strength is in $\mathrm{V} / \AA(1 \mathrm{~V} / \AA=10 \mathrm{GV} / \mathrm{m})$.

Table I gives the parameters for a copper tip and the critical electric field $F^{1+}$ that is necessary to evaporate a single charged ion obtained from Eq. (1).

Müller's model postulates that escape for a surface atom occurs when it has a well-defined ion charge state before and after it overcomes the potential barrier [9]. This mechanism gives an Arrhenius-type rate constant, which could well be compared to experiments at temperatures below $80 \mathrm{~K}$ [18].

TABLE I. Parameters for copper tip and critical electric field $F^{1+}$ needed to evaporate single charged ion obtained from Eq. (1).

\begin{tabular}{cccccccc}
\hline \hline $\begin{array}{c}\Lambda \\
(\mathrm{eV})\end{array}$ & $\begin{array}{c}\phi \\
(\mathrm{eV})\end{array}$ & $\begin{array}{c}r_{0} \\
(\AA)\end{array}$ & $\begin{array}{c}I_{1} \\
(\mathrm{eV})\end{array}$ & $\begin{array}{c}I_{2} \\
(\mathrm{eV})\end{array}$ & $\begin{array}{c}I_{3} \\
(\mathrm{eV})\end{array}$ & $\begin{array}{c}E_{\mathrm{aff}} \\
(\mathrm{eV})\end{array}$ & $\begin{array}{c}F^{1+} \\
(\mathrm{GV} / \mathrm{m})\end{array}$ \\
\hline 3.50 & 4.60 & 1.25 & 7.73 & 20.29 & 36.83 & 1.23 & 30 \\
\hline \hline
\end{tabular}


Equation (1) could further be modified if one takes into account atomic and ionic polarizability that may induce a noticeable polarization effect and contribute to the activation energy of field desorption [19]. In Ref. [20] the activation energy of individual atoms has been shown to depend on electric field strength.

A few other DCFE models were discussed in Refs. [1921]. None of these models is applicable to evaporation at room and higher temperatures. The escape mechanisms at high temperatures should include surface diffusion of atoms that cover the tip and the vicinity close to the tip [22]. Therefore, the existing DCFE theory cannot predict the critical field and evaporation flux at elevated and high temperatures.

Field evaporation at an rf field (RFFE) was not studied before in FIM experiments because an rf field is unable to create surface imaging. However, the FIM samples were pulsed with 1 ns pulses and could be related to RFFE.

Therefore, we believe that this is the first effort to clarify whether or not field evaporation effect may be considered important in an rf cavity as a candidate mechanism for the triggering of rf- vacuum breakdown.

\section{MOLECULAR DYNAMICS SIMULATION OF FIELD EVAPORATION}

To atomistically simulate field evaporation, we have used a classical MD method that is capable of revealing temperature effects. In this MD method, the equations of motion for interacting particles are solved numerically and appropriate initial and boundary conditions are applied [23].

To simulate field evaporation of a metal tip on an rfcavity surface, all tip atoms and a "reasonably large" number of surface atoms are treated in detail as "mobile," while the rest of the target is represented by a thermostat. The mobile atoms, with more violent behavior like diffusion, evaporation, or sticking, are studied by solving the set of exact Newton's equations of motion, for which classical forces are obtained from proper interatomic potentials. Two more forces were applied to the charged tip particles: one force was acting on behalf of a periodic rf field and the other was describing the image forces acting on a charge near the metal surface.

The thermostat was modeled by using the continuum mechanics elasticity equations and linear thermodynamics. The details of our MD method are described elsewhere [8].

In the MD calculations, we used a $\mathrm{Cu}$ (100) surface on the top of which was placed a bell-shaped tip that was simply cut of a face centered cubic $\mathrm{Cu}$ lattice. The $\mathrm{Cu}$ atoms of the mobile zone were interacting via a manybodied embedded-atom method potential derived from a second-momentum approximation of the tight binding scheme. The following set of parameters was used for the potential: $E_{c}=3.50 \mathrm{eV}, Z_{\mathrm{nn}}=12, p=10.08, q=2.56$, where $E_{c}$ is the lattice cohesion energy, $Z_{\mathrm{nn}}$ is the number of nearest neighbors, and $p$ and $q$ are the potential function exponents. This choice of parameters has been devised to attain correct dynamics at room and elevated temperatures [24]. The cylindrical surface model contained $\approx 10^{4}$ atoms in the central MD zone, while the continuum mechanics calculations extended to a volume that was many times larger.

Image forces (Schottky barrier forces) were applied to the charges that were on the top of the tip; the number and location of the charges was obtained from the condition that included the tip's curvature and number of neighbors. ${ }^{1}$

An additional periodic electric field was applied to the charges on the surface of the tip: $\boldsymbol{E}(t)=\boldsymbol{E}_{0} \sin (\boldsymbol{\omega} t)$. Here $\boldsymbol{E}_{0}$ is the maximum value, $\boldsymbol{\omega}$ the angular frequency, and $t$ is the time elapsed from the beginning of the evaporation process.

Our preliminary study has shown that the magnet force acting to the tip's atoms does not change the critical fields of breakdown. Therefore they were not taken into account at this time. More details on the effect of magnet field could be found in a previous paper [7].

Three frequencies of the rf field were modeled: 600 and $800 \mathrm{MHz}$ and $1.2 \mathrm{GHz}$, which correspond to existing linacs. The maximum computation time $t_{m}$ was then defined as one quarter of the period of rf-field oscillation, such as $t_{m}=T / 4=1 / 4 f$, where $f$ is the frequency of the field. Therefore, for $f=600 \mathrm{MHz}, T=1 / f=1.66 \mathrm{~ns}$, and computation time was $t_{m}=414 \mathrm{ps}$. This parameter was $312 \mathrm{ps}$ for the frequency of $800 \mathrm{MHz}$ and $200 \mathrm{ps}$ for the frequency of $1.25 \mathrm{GHz}$. Our computations started at a zero phase and continued up to lowest phase of $\pi / 2$.

The $\mathrm{Cu}$ tip was modeled by $200-1000 \mathrm{Cu}$ atoms built as a bell, with bottom diameters of $\approx 54-125 \AA$ and heights of 15-30 ̊̊ above a Cu (100) surface. Bigger tips were also modeled for comparison.

\section{MOLECULAR DYNAMICS RESULTS FOR A COPPER TIP}

Various temperatures, from room up to the melting point of bulk $\mathrm{Cu}$, and local electric field gradients of $1-100 \mathrm{GV} / \mathrm{m}$ were modeled. Evaporation of groups that contained between 10 and $200 \mathrm{Cu}$ atoms and ions was simulated for the first time. According to the results, the vacuum inside the high-gradient rf cavity should contain a noticeable presence of nanometer-size chunks that evaporated from various intrusions that exist on the real cavity surface by an rf field.

\section{A. Room temperature results}

Figure 1(a) shows the initial position of a $\mathrm{Cu}$ tip placed on the top of a $\mathrm{Cu}$ (100) surface. After the tip and the surface were equilibrated for $20 \mathrm{ps}$ without an electric field,

\footnotetext{
${ }^{1}$ The total number of charges per tip was estimated by Gauss's Law for the field of $10 \mathrm{GV} / \mathrm{m}$ and the tip's diameter of $100 \AA$. The absolute charge per ion was $q_{i}=+e \sin (\omega t)$; i.e., it was periodic with the field frequency.
} 

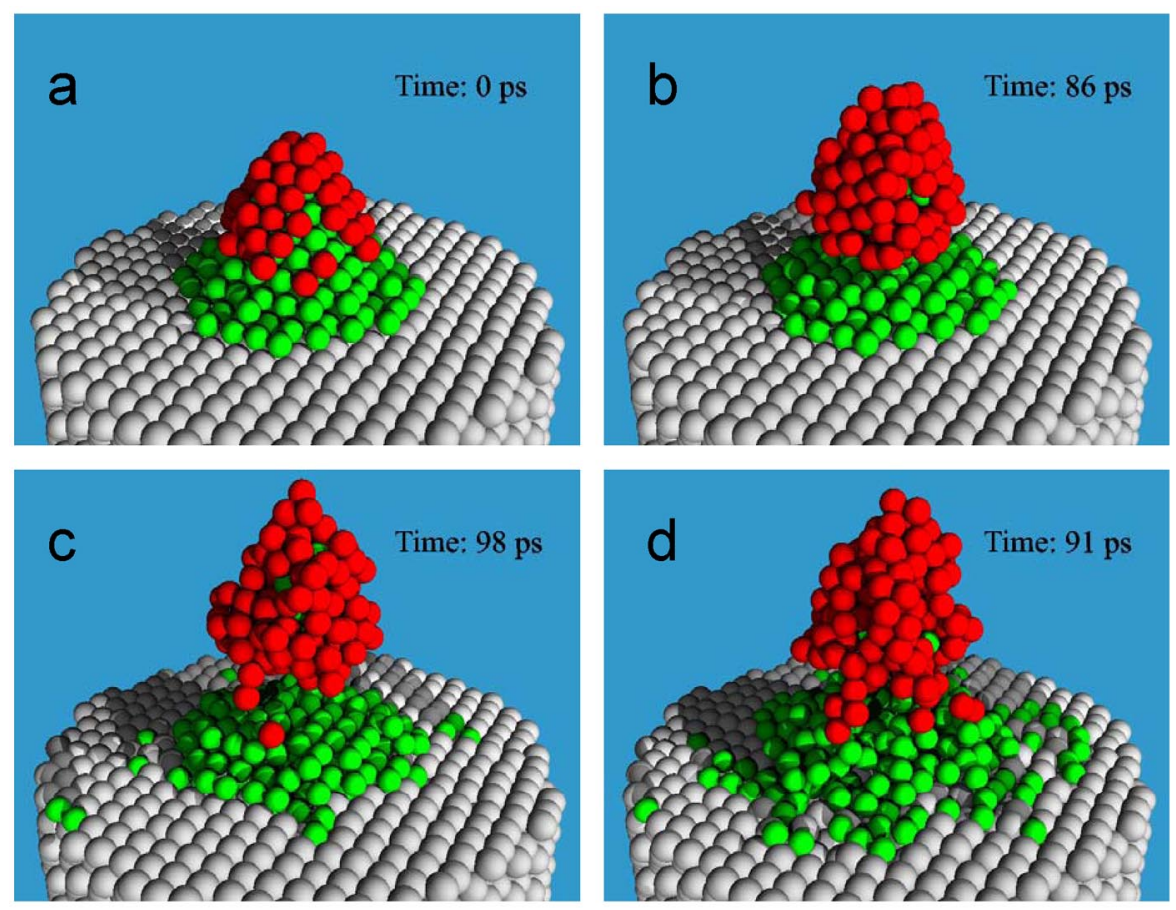

FIG. 1. (Color) (a) Initial view of a $\mathrm{Cu}$ tip. Red: ions; green: neutral tip atoms; gray: the atoms of a Cu (100) surface that have no electric charge. (b) $\mathrm{Cu}$ tip after $100 \mathrm{ps}$, in rf field of $E_{m}=10 \mathrm{GV} / \mathrm{m}$, frequency of $f=1.25 \mathrm{GHz}$, at $T=545 \mathrm{~K}$. (c) $10 \mathrm{GV} / \mathrm{m}$, $T=990 \mathrm{~K}$, and $1.25 \mathrm{GHz}$. (d) $\mathrm{Cu}$ tip for $10 \mathrm{GV} / \mathrm{m}, 1.25 \mathrm{GHz}$, at temperature of $1300 \mathrm{~K}$. Time instants are shown in figures.

the MD simulation was followed up to $200-400$ ps depending on the frequency of the rf field, with periodic electric and image forces applied to part of the tip's particles. The time evolution of the tip under applied rf field and the dynamics of the tip's atoms were followed by MD. A movie was made for various temperatures and field magnitudes.

Our results at an rf-field magnitude of $1 \mathrm{GV} / \mathrm{m}$ and frequency of $1.25 \mathrm{GHz}$ at room temperature show significant diffusion motion and significant atomic rearrangement of the tip's atoms. However, no significant changes were observed in the tip's geometry, i.e., no particles were evaporated for times up to $200 \mathrm{ps}$, the maximum time for the simulation, chosen to be equal to a quarter of the oscillation period of the applied rf field.

When the field is increased to $30 \mathrm{GV} / \mathrm{m}$, and the frequency of the electric field is dropped to $800 \mathrm{MHz}$, the tip starts to evaporate at $100 \mathrm{ps}$. After $\approx 300 \mathrm{ps}$, the top of the tip is pulled out completely and moved into the vacuum. The surface of the tip becomes much smoother after some part of the tip is removed.

This means that an electric field that does not detach and evaporate the nanotips on the cavity surface at a higher frequency of the rf field would do that more easily at a lower frequency field or in a dc field. Therefore, the results obtained by experiments in a dc field are not immediately applicable to the rf cavity study; they should be done separately.

Cluster evaporation might have a significant effect in FIM experiments [12]. However, because this event had always been deemed a tip failure, it had not been studied in detail.

Inasmuch as our observation was obtained for an rf field, this is a new field evaporation effect that might also be a new rf-vacuum breakdown triggering mechanism. Although it has never been observed before [25], the new effect is similar to the foreign microparticle or "Cranberg" mechanism [1].

\section{B. Elevated temperatures: $T=545-650 \mathrm{~K}$}

Figure 1(b) shows the dynamics of the $\mathrm{Cu}$ tip at $545 \mathrm{~K}$, with applied electric field maximum value of $10 \mathrm{GV} / \mathrm{m}$ and $1.25 \mathrm{GHz}$ frequency. The time instant corresponds to 86 ps: a field evaporation of a large group of atoms. This result is easy to understand from force balance: the surface cohesion forces could be stronger than the electric force for a single atom or for a small group of atoms. However, the same electric field would evaporate a larger group of ions because the total electric force is linearly proportional to the number of ions in the group. Because electric charge density strongly depends on surface curvature, it will be a balance between the electric and cohesion forces and our simulation shows that there are between 100 and 300 ions in the cluster.

\section{High-temperature simulation results: $T=\mathbf{8 0 0}-1300 \mathrm{~K}$}

The effect of the surface temperature has been studied in simulations at increased tip temperatures from $800 \mathrm{~K}$ and 
up to the melting temperature of bulk $\mathrm{Cu}$, in electric fields from 1 to $100 \mathrm{GV} / \mathrm{m}$. Our simulation at $800 \mathrm{~K}$ shows that field evaporation has started at an earlier time instant of $50 \mathrm{ps}$, compared with the previous case at $545 \mathrm{~K}$ where the detachment occurred at 86 ps. After a large cluster was removed from the tip, small fragments are leaving the tip with the same velocity.

The green atoms were identified as those with a vertical position above the initial surface level, which was at $x=0$. Removal of the tip leads to an interesting phenomenon, in which other surface atoms acquire elevated positions above the surface. Figures 1(c) and 1(d) show a few green surface atoms, with higher vertical positions. Such a process could lead to formation of new tips and new evaporation events, even if they did not exist before the electric force was applied. The number of green atoms is clearly increasing with temperature. This simulation result is in agreement with more fundamental properties of a solid or liquid surface under an applied electric force. It has been known for a long time that such forces could change the shape of the tip and even lead to the growth of a whisker [25].

Figure 1(d) presents the simulation result obtained at a temperature close to the melting point for bulk $\mathrm{Cu}, 1311 \mathrm{~K}$ [26]. The applied electric field was $10 \mathrm{GV} / \mathrm{m}$, with a frequency of $1.25 \mathrm{GHz}$. We see that temperature has a striking effect. Figure 1(d) shows that after 91 ps the shape of the tip has dramatically changed and the top of the tip is ready to escape into vacuum.

In Fig. 2, the critical electrical strengths at which a large cluster starts to detach from the body of the tip were plotted for five temperatures, starting from room temperature and up to the melting point of bulk.

The obtained critical electric fields are much lower than that for a single-ion evaporation of $30 \mathrm{GV} / \mathrm{m}$ (Table I).

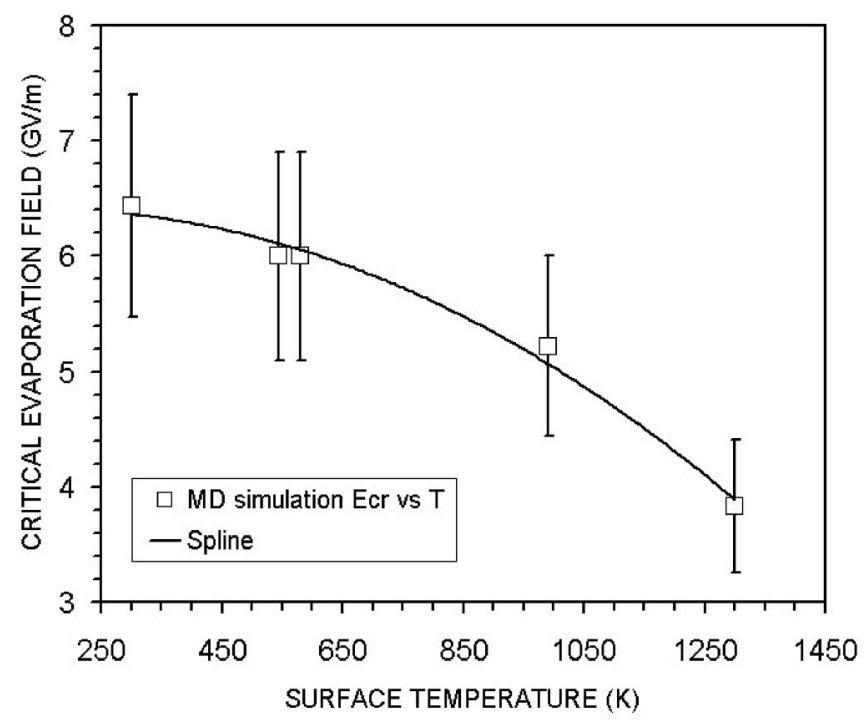

FIG. 2. Observed temperature dependence of critical evaporation field for removing cluster of $\approx 200 \mathrm{Cu}$ ions.
This means the field evaporation would most probably occur by cluster evaporation, rather than by single ions.

\section{COMPARISON WITH EXPERIMENT}

Experiment [5] on the vacuum breakdown in rf field shows that the breakdown is almost independent of cavity temperature. Our results shown in Fig. 2 also predict a rather weak temperature dependence of the critical field. There is no experimental work done in finding cluster evaporation in rf field. However, this phenomenon is well studied for dc-electric fields. Figure 3 shows abrupt discontinuities in the voltage versus number of ions in a field evaporation system and shows evidence for large clusters produced at field ion microscope tips [27]. The numbers at the discontinuities show the radii and the sizes of clusters evaporated from the tip. The approximate size of the fragments can be estimated from the size of the discontinuity and the density of the material.

The results show a failure of the tip because an image cannot be obtained by such a tip, and therefore, it is an undesirable event.

Our model predicts that single ions have less probability to be field evaporated in a high-gradient rf field. However, this does not mean that they will not be evaporated in a high-gradient dc-electric field. Experiment shows that at low-temperature field desorption, field-evaporated ions are almost always atomic ions [12]. On the other hand, in pulsed-laser-simulated field evaporation experiments, many cluster ions were detected [28]; the amount could be as high as $30 \%$ of the total evaporated ions. Our model could easily be adjusted to study a dc-field field evaporation and this work is under way.

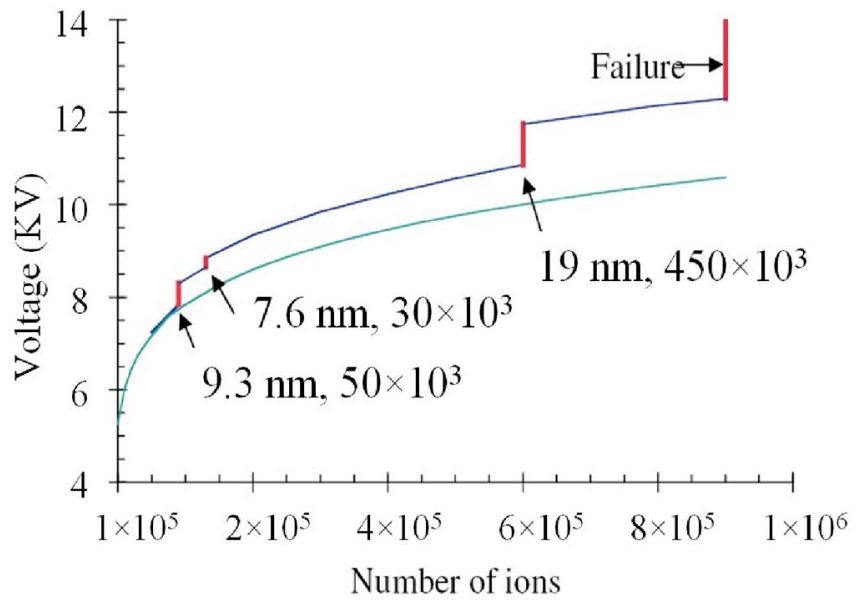

FIG. 3. (Color) Abrupt discontinuities in the voltage versus number of ions in a field evaporation system show evidence for large clusters produced at field ion microscope tips, from Ref. [27]. The approximate size of the fragments can be estimated from the size of the discontinuity and the density of the material. 
Coulomb explosion of field-evaporated highly charged cluster ions, interaction of such clusters with the rest of the surface, and heating of clusters by dark-current electron emission would significantly contribute to the understanding of the vacuum breakdown triggering mechanism and they will be the subjects of further improvement of the MD model.

There is a need for specific experiments to detect, observe, and study such clusters to verify the new mechanism of vacuum breakdown studied in this paper.

\section{CONCLUSION}

We have successfully simulated field evaporation of large clusters of atoms and/or ions from a nanometer-size $\mathrm{Cu}$ tip placed on top of a $\mathrm{Cu}$ cavity, within a strong rf field applied to the surface. Various electric fields, frequencies, and surface temperatures were modeled. A critical electrical evaporation field was obtained for temperatures that range from room up to the melting point of bulk $\mathrm{Cu}$. The simulation results were compared with available data on FIM tip fracture in a dc electric field.

[1] L. Cranberg, J. Appl. Phys. 23, 518 (1952).

[2] J. Knobloch, Ph.D. thesis, Cornell University, 1997.

[3] L. L. Laurent, Ph.D. thesis, Stanford University, 2002.

[4] W. Dyke and J. K. Trolan, Phys. Rev. 89, 799 (1953); W. P. Dyke, J. K. Trolan, E. E. Martin, and J. P. Barbour, Phys. Rev. 91, 1043 (1953).

[5] H. H. Braun, S. Dobert, I. Wilson, and W. Wuensch, Phys. Rev. Lett. 90, 224801 (2003).

[6] J. Norem et al., Phys. Rev. ST Accel. Beams 6, 072001 (2003).
[7] J. Norem, Z. Insepov, and I. Konkashbaev, Nucl. Instrum. Methods (to be published).

[8] Z. Insepov et al., Phys. Rev. B 61, 8744 (2000).

[9] E. W. Müller, Phys. Rev. 102, 618 (1956).

[10] E. W. Müller and T. T. Tsong, Field Ion MicroscopyPrinciples and Applications (American Elsevier, New York, 1969), p. 56.

[11] T. T. Tsong, Jpn. J. Appl. Phys. 30, L1985 (1991).

[12] T. T. Tsong, Atom-Probe Field Ion Microscopy (Cambridge University, Cambridge, England, 1990), p. 56.

[13] D. G. Brandon, Br. J. Appl. Phys. 16, 683 (1965).

[14] C. S. Chang, W. B. Su, and T. T. Tsong, Phys. Rev. Lett. 72, 574 (1994).

[15] N. M. Miskovsky, C. M. Wei, and T. T. Tsong, Phys. Rev. Lett. 69, 2427 (1992).

[16] N. M. Miskovsky and T. T. Tsong, Phys. Rev. B 46, 2640 (1992).

[17] T. T. Tsong, Phys. Rev. B 44, 13703 (1991).

[18] R. G. Forbes, Appl. Surf. Sci. 87/88, 1 (1995).

[19] T. T. Tsong, J. Chem. Phys. 54, 4205 (1971).

[20] G. L. Kellogg, Phys. Rev. B 29, 4304 (1984).

[21] D. N. Zhulev and R. G. Forbes, J. Phys. D 36, L74 (2003).

[22] V. N. Shrednik, D. V. Glazanov, and E. L. Kontorovich, Tech. Phys. 48, 1199 (2003).

[23] D. Frenkel and B. Smit, Understanding Molecular Simulation (Academic, New York, 1996).

[24] V. Rosato, M. Guillope, and B. Legrand, Philos. Mag. A 59, 321 (1989).

[25] G. K. Wehner, J. Vac. Sci. Technol. A 3, 1821 (1985).

[26] C. Kittel, Introduction to Solid State Physics (Wiley, New York, 1986), 6th ed., p. 55.

[27] M. K. Miller, Atom Probe Tomography, Analysis at the Atomic Level (Kluwer Academic/Plenum, New York, 2000).

[28] K. Sattler, J. Mühlbach, O. Echt, P. Pfau, and E. Recknagel, Phys. Rev. Lett. 47, 160 (1981). 